\title{
Research on Packaging Design of Take-Out Food Oriented Towards the Usability Enhancement
}

\author{
Guang YANG \\ Suzhou Vocational University, Suzhou, China
}

\begin{abstract}
Usability enhancement of take-out food packaging in its whole life circle was analyzed in this paper from the standpoint of businesses, shop assistants, consumer and environment, respectively. Results indicated that usability enhancement of take-out food packaging can be achieved by taking following measures: producing universal packages as soon as possible, reducing packaging decoration design, decreasing space occupation by using foldable packages, designing self-sealing and auto end-locked structure to facilitate enclosing and opening of packages, reducing quantity of packaging materials. These results provide packaging designers (or more broadly, design decision makers) with more advanced design concepts in the process of take-out food packaging design.
\end{abstract}

KEYWORD: Usability; Take-out food packaging; Process; Design

\section{INTRODUCTION}

Usability as an interdisciplinary subject has been obtained more attention in information science, library science, software engineering, humanmachine interaction and other fields in recent decades [1]. Commonly accepted definition of usability is that defined in ISO 9241-11, in which the usability is defined as the extent to which a product can be used by specified users to achieve specified goals with effectiveness, efficiency and satisfaction in a specified context of use. In this definition, effectiveness means accuracy and completeness to accomplish the target specification, efficiency denotes the depletion of resources in order to attain those goals precisely, while satisfaction means the comfortable degree and the acceptance of consumer [2].

As a kind of package having specific function, take-out food package is somewhat different from that for window display, because users of the former package include not only consumers carrying and using it, but also the shop assistants packed for consumer. Thus, the efficiency of packing process will be an important factor in consumer satisfaction improvement. Therefore, relevant elements of package design are studied in this paper, with the aim to explore methods to improve the usability of take-out food package.

\section{WHOLE LIFE CIRCLES OF TAKE-OUT FOOD PACKAGE}

Generally, interaction between consumer and package can be roughly divided into three stages: obtain package, uses it, and finally deal with package waste [3]. Different from ordinary package, the interaction process of take-out food package includes only two stages, because purchasing behavior has already happened before consumer obtained take-out food package. In other words, it is passive for consumer to obtain package, the shop assistants are the dominant part in first stage; it is very different from shelf display product which having visual impact to inspires consumer's impulse shopping. In next stage, the shop assistants packed take-out food for consumer, and they are main participant of this process while consumers will not participate in. However, consumer in waiting state may affect pack operation of the shop assistants, for they hope that the waiting time can be shortened and package operation accomplished more efficiently. Then, consumers obtained their take-out foods, carry them, eat them and then discard packages, consumers are main participant in this stage. Finally, these discarded packages are recycled or destroyed and enter into the environment eventually.

Therefore, from above processes, the businesses, shop assistants, consumers and environment constitutes the "specified users" in the definition of ISO 9241-11. Packages are produced by package 
supplier, shop assistants pack for consumer, consumer use and discard package, the environment assimilate these waste, and thus these four interconnected processes constitute the "specified contexts" in the definition. Now we will analyze relevant elements in usability enhancement of takeout food package from the specified contexts:

\subsection{From the standpoint of the businesses:}

Doggy bag is a kind of service provide by the businesses to facilitate consumer, they are free or charge at a lower rate. This fact remains that cost become a very important factor in selecting take-out food package, i.e., the businesses choose packages that can meet the basic functions with lowest cost. These basic functions included segregates different food in one package, protects against any spillage of liquid food, supply complementary straw, spoon and paper napkins. Based on the cost minimization strategy, we can see that common used packaging materials are cheaper materials such as paper and plastic, while metal and glass are rarely used for their expensive price. Although the latter has better packaging performance than the former and can satisfy additional functions such as reheating directly in a microwave oven and getting great heatpreservation property. Therefore, from the perspective of the businesses, design factors to improve the usability of take-out food package are as following:

\subsubsection{Design and produce universal packages as soon as possible}

There are a rich variety of foods with different sizes, thus we need all kinds of package with different specification to fit all foods. However, this is often an impractical task because of the cost and the capital occupation. Therefore, the businesses should categorize foods and design universal package for one specified category, by this way they can reduce unnecessary cost.

\subsubsection{Reduce packaging decoration design}

A beautifully printed and bright-colored package has always been an effective vehicle for advertising. However, paper is a type of permeable material and the application of edible ink is far from mature. Thus, printing ink may squeeze into foods, the larger the print area, the more the infiltrations [4]. From the standpoint of food safety, take-out food package should reduce packaging decoration and advocate healthy diet concept.

\subsection{From the standpoint of the shop assistants}

Effectiveness and efficiency of packing process should be emphasized when shop assistants packed for consumer. They picked doggy bag, put the foods purchased by the consumer into the bag, seal the bag and hand it to consumer. This whole series of actions takes place when consumers are waiting, therefore, the shop assistants should packed rapidly and efficiently in order to reduce the waiting time. While a rational and effective structural design of package will greatly increase efficiency. Therefore, from the perspective of the shop assistants, design factors to improve the usability of take-out food package are as following:

\subsubsection{Reduce space occupation and facilitate pick action through structural design}

Empty cartons occupying valuable counter space, thus cartons that can be flattened and refolded are recommended. It should be noted that stacked flattened cartons are more apt to adhere to each other. When we pick one carton, there are multiple cartons, so we should replace unnecessary cartons on the shelf. Obviously, this extends the waiting time and ultimately undermines the effectiveness. Thus, this problem should be avoided in the stage of packaging structural design.

\subsubsection{Facilitate rapid sealing through structural design}

It is preferred that sealing of take-out food package achieved by structural design of package itself. That is, self-sealing structural designs such as auto endlocked structure and tenon and mortise joint designed for paper carton will be best. Figure 1 illustrates an auto end-locked carton, this carton can flatten and refold easily, and the bottom of carton can lock automatically during refolding process. Similarly, zipper settled on the opening of plastic bag is a good idea to seal the bag conveniently.

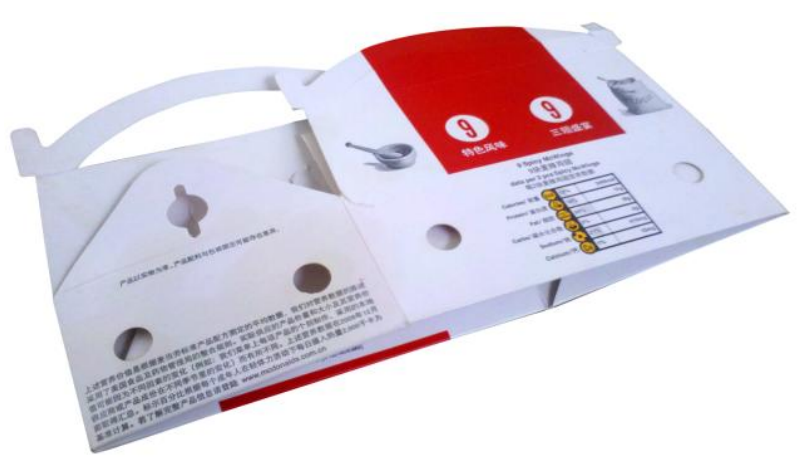

Figure 1. Structural design of auto end-locked carton.

\subsection{From the standpoint of consumers}

Once consumers access the take-out food, they will carry the package, open it, enjoy delicious food and then ditched the package waste. Thus, focus of packaging usability investigation will transfer to consumers. Therefore, from the perspective of consumers, design factors to improve the usability of take-out food package are as following: 


\subsubsection{Portability of packages}

Designers should constantly imagine themselves to be in the consumers' position to make packages conveniently in every detail. For example, whether there are handles for consumers to carry by a single hand rather than hold with both hands; whether packages can be suspend or not; whether the bottom of package is designed to be flat in order that consumers can put them on the back seat of car, and whether it is conveniently for consumer to placed packages on the ground directly to open the door. Such details should be considered during packaging design.

\subsubsection{Safety opening structure design}

Packages should be opened easily, without tools such as scissors and razor blades. One thing to keep in mind is that tiny parts of packages should avoid to be swallowed by children after you open it. Meanwhile, it is more conveniently to design the opening structure that can re-sealed.

\subsubsection{Information cue of potential unsafe factors}

As shown in Figure 2 (a), some fried foods such as pie have very hot fillings which may burn consumer's mouth, thus this factor should be taken into account in packaging design to reminding and warning consumers. Such potential unsafe factors may include sucking hot drink through a straw, eating wrapping paper by mistake because the color of wrapping paper is very close to the color of food and the potential hazard of asphyxiation caused by put plastic bags over children's head (Figure 2 (a)). These potential unsafe factors should be printed on the packages to give consumers more direct and clear warnings.

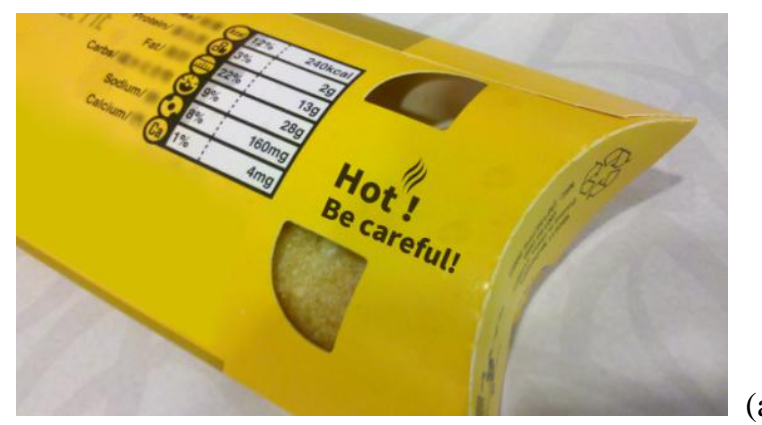

(a)

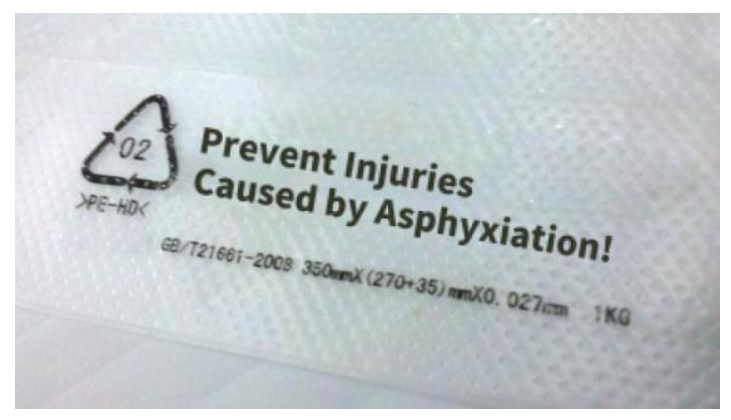

(b)

Fig. 2 Indicative design about potential insecurity

\subsection{From the standpoint of environment}

Environmental impact of packaging waste is often ignored by many designers. However, in the context of low-carbon economy, packaging designers needs not only innovation at a technical level but reformation of concept. Thus, product's functions, visual aesthetic effects, market purchasing power and environmental benefits should be considered and designed with the perspective of package life-cycle in mind to achieve harmony of economic returns and contribution to society and environmental protection [5]. Therefore, from the perspective of the environment, design factors to improve the usability of take-out food package are as following:

\subsubsection{Choose appropriate packaging materials}

Materials with minimum environmental burden and higher recycle utilization rate are preferred, such as paper products, plant-fiber products, biodegradable plastic, edible packaging materials, etc. Try to avoid using packaging materials having a surface coating or Plating, because the coatings are hardly be separated from the base material, which may increase the treatment difficult of waste.

\subsubsection{Design concept of packaging reduction}

On the premise that the gap between the content and package keep at a reasonable level, designers can obtain maximum ratio of content weight to material weight through innovative structural design, subsequently achieve reduction design. For example, octagonal pizza carton will reduce the use of packaging materials by 10 per cent than square box, and correspondingly reduce carbon emissions by 10 percent.

\section{CONCLUSION}

Design factors to improve the usability of take-out food package were analyzed in this paper from the perspective of businesses, shop assistants, consumers and environment. Designer involvement in usability studies should bear the whole life cycle of package in mind, and to improve the usability of take-out food package under the theory of nature and humanity. Only in this way can we make life better, achieve harmony between economy and environment, and promote the all-round development of our society.

\section{REFERENCES}

[1] WANG Jian-dong. 2009. A Literature Review of Progress in Foreign Usability Research. New Technology of Library and Information Service, (09):7-16. 
[2] Swann M. 2009.Ergonomic Requirements for Office Work with Visual Display Terminals (VDTs). [2009-0717].http://www. ergonomicsolutions.net/pdf/ISO9241Summary.pdf

[3] GE Lie-zhong, WAN Jia. 2010. Review of Packaging Usability Research. Packaging Engineering, 31(3):132136.
[4] LIU Guang-fu, LIU Zhi-feng, LI Gang. 2004. Green Design and Green Manufacturing. Beijing: China Machine Press,.

[5] YANG Guang, E Yu-ping. 2011. Packaging Design in Low-carbon Time. Packaging Engineering 32(4): 81- 83. 